\title{
First Evaluation of Human Sperm Quality in Various Geographic Regions of Switzerland
}

\author{
Michel Crausaz ${ }^{\star a}$, Josefina Vargas ${ }^{\mathrm{a}}$, Roumen Parapanov ${ }^{\mathrm{a}}$, Yves Chollet ${ }^{\mathrm{b}}$, Marc Wisard ${ }^{\mathrm{bc}}$, Eric \\ Stettler ${ }^{\mathrm{d}}$, Alfred Senn ${ }^{\mathrm{ac}}$, and Marc Germond ${ }^{\mathrm{ac}}$
}

\begin{abstract}
A decline in human sperm quality and quantity has been reported in numerous Western countries. This observation was also accompanied by an increase in urogenital malformations. The need for epidemiological studies dealing with unbiased populations in order to understand the causes of these observations is obvious. In Switzerland, the large majority of young men are asked to attend a military camp to be drafted into the army. A few weeks before this camp, conscripts were contacted and invited to participate in a large national study on semen quality. The participation was totally voluntary and anonymous. From September 2005 to June 2007, 770 volunteers filled out a questionnaire, underwent a clinical examination and provided sperm, blood and urine samples. Using self-rated health assessments, the observed cohort could be considered as healthy and no testicular cancer was found. Moreover, the testicular volumes, measured using Prader's orchidometry and ultrasonography, were comparable to those already published for young male populations. The median sperm concentration was $47 \times 10^{6} / \mathrm{ml}$, which is close to the concentration reported in Denmark, known to have the highest incidence of testicular cancer in Europe. Statistically significant differences were observed between regions with a lower sperm concentration for men residing in the Alps $\left(43 \times 10^{6} / \mathrm{ml}\right)$ and in the Zürich area $\left(36 \times 10^{6} / \mathrm{ml}\right)$ compared to men from West Plateau $\left(54 \times 10^{6} / \mathrm{ml}\right)$ and from the Jura $\left(54 \times 10^{6} / \mathrm{ml}\right)$. Such a regional discrepancy could be related to environmental factors, including endocrine disruptors. In order to confirm such regional differences more volunteers from the already studied regions should be studied and other parts of the country should be investigated. The rather low sperm concentration of Swiss young volunteers should be considered as a national health issue and investigated further.
\end{abstract}

Keywords: CASA $\cdot$ Epidemiological study $\cdot$ Sperm count $\cdot$ Semen $\cdot$ Switzerland

\section{Introduction}

Over the past few decades, various animal species have started to show abnormalities in their reproductive system. ${ }^{[1-3]}$ One of the best-known examples concerns the al-

${ }^{*}$ Correspondence: M. Crausaz ${ }^{a}$

Tel.: +41213435150

Fax: +41213435159

E-mail: michel.crausaz@fondation-faber.ch

aF.A.B.E.R. Foundation

Rue de la Vigie 5

$\mathrm{CH}-1003$ Lausanne

bepartment of Urology

Centre Hospitalier Universitaire Vaudois

$\mathrm{CH}-1011$ Lausanne

${ }^{\circ} \mathrm{CPMA}$, Avenue Marc-Dufour 1

$\mathrm{CH}-1007$ Lausanne

dSwiss Armed Forces Joint Staff - Medical Services Worblentalstrasse 36

$\mathrm{CH}-3063$ Ittigen ligators from the Apopka Lake in Florida. An abnormal ovarian morphology as well as disorganized testes and penis size were reported. ${ }^{[4]}$ In a recent study comparing the development of young alligators in two American lakes, such deteriorations were correlated to a maternal exposure to certain chemicals, such as dichlorodiphenyltrichloroethane (DDT). ${ }^{[5]}$ Another example is the imposex phenomenon. The masculinisation of marine gastropods was linked to infinitesimal doses $(<0.4 \mathrm{ng} / \mathrm{l})$ of tributyltin (TBT), used since the 1960s as an antifouling agent. ${ }^{[1]}$ In the Lake Thun, Switzerland, morphologic alterations of the gonads were observed in $35 \%$ of whitefish (Coregonus $\mathrm{sp})$. The causes have not yet been discovered. ${ }^{[6]}$ In some rivers of the Swiss Plateau receiving sewage effluents, brown and rainbow trout have altered gills, liver and kidneys leading to a mortality increase. ${ }^{[7]}$

The precise causes of these defects and malfunctions are still poorly understood. An assumption often advanced is the possible implication of endocrine disruptors. These chemicals, biologically active at very low doses, can mimic or alter the ac- tions of endogenous hormones and thereby unbalance the endocrine system, inducing harmful effects on living beings and their offspring. ${ }^{[8]}$

In humans, the first identified endocrine disruptor was diethylstilbestrol (DES). This synthetic, non-steroidal oestrogen was used from the1940s to 1970s in the United States and France for pregnant women to prevent spontaneous abortions. In the 1960s, the first cases of vaginal adenocarcinoma emerged in girls exposed in utero to DES. [9] Very quickly, it was found that other urogenital abnormalities among women and men could be linked to DES exposure. ${ }^{[10]}$ Testicular dysgenesis syndrome (TDS), including cryptorchidism (testicular maldescent), hypospadias, reduced spermatogenesis and testicular cancer, has often been observed in men exposed to DES during foetal life. ${ }^{[11]}$ The etiology of TDS is suspected to be associated with environmental factors, in particular endocrine disruptors. For obvious ethical reasons, the experimental study of their effects in humans is impractical. However, as one of the manifestations of TDS is the decrease of sperm 
density, monitoring this parameter could indirectly enable the detection of disruptive substances in the environment.

The increase, in recent decades, of endocrine disruptors in the environment could be responsible for the decrease of the sperm concentration in human. Although some studies indicate no change in the number of sperm produced by human males during the past 20 years, ${ }^{[12]}$ others suggest a decrease ${ }^{[13]}$ accompanied by an increase of hypospadias, cryptorchidism and testicular cancers. ${ }^{[14]}$ Several studies have established links between sperm quality and years of analysis, the year of birth ${ }^{[15]}$ or the geographic region of residence. ${ }^{[16]}$ In a metaanalysis combining data from 101 studies published between 1934 and 1996, Swann et al. ${ }^{[13]}$ highlighted, among men in North America and West Europe, a decline in sperm concentration as a function of time.

At this point, the biological mechanisms of action to explain the decline of sperm production in humans are not well known and are reduced to a series of assumptions. One of them established a link between foetal exposure to certain chemicals and the onset of fertility disorders during adult life. Storgaard et al. showed that the quantity of cigarettes smoked by the mother during pregnancy can affect the sperm quality of the child. [17] Other more direct links between the environment and human fertility have been raised. In the United States, men living in an agricultural state (Missouri) have a lower sperm concentration than men living in Minnesota, a region less exposed to phytosanitary chemical compounds. ${ }^{[18]}$

Some of the published retrospective studies have attracted criticisms by experts. ${ }^{[16]}$ A need for prospective studies collecting a large number of parameters in an unbiased population has become necessary in order to confirm a correlation between the sperm concentration decrease and the environment.

Our study on male fertility, which is part of the NRP50 program, was designed to evaluate environmental influences on sperm quality and on urogenital tract integrity in young men from various regions of Switzerland. In a decade, it is planned to re-contact each participant to obtain data on fecundity and general health. Here, we report preliminary results on the sperm quality of a cohort of young Swiss volunteers at the time they are drafted by the Swiss Army.

\section{Materials and Methods}

\section{Studied Population}

All healthy Swiss men aged between 18 and 20 are invited for a three-day camp in order to be drafted into the Swiss Army. Due to the fact that only men without di- agnosed chronic diseases are contacted, the population of conscripts represents $95-96 \%$ of the total Swiss young men (ES, personal communication). The aim of our study is to collect data on 3000 volunteers all around the country. In order to avoid over-representations, a quota for each canton was calculated according to the total population density. When this quota was reached for a canton, the enrolment of cases was stopped for this region.

The procedure adopted for this study can be summarized as follows. One month before the camp, each conscript received an information letter explaining the goals of the study, a consent form and two different questionnaires, one for himself and one for his parents. If he agreed to participate, the conscript filled out and sent back his documents. The parents handled their questionnaire separately. All documents were addressed to the Swiss Army physician (ES), who was in charge of the study in the military department and ensured the volunteers' anonymity. Once the military recruitment was over, the volunteers were asked to stop on their way back home at our main laboratory in Lausanne or in the associated centres (Rüti, Monte Ceneri) in order to go through the clinical and biological investigations of the study. The whole procedure was approved by the Ethical Committees of Lausanne, Zürich and Ticino.

\section{Questionnaires}

Each volunteer completed a questionnaire including not only general data (year of birth, region of residence, current height and weight) but also information on health (self-rated health, temperature, drug intake), lifestyle factors (smoking habits, alcohol intake, narcotic consumption, diet), education and occupation. Detailed aspects on previous urogenital history such as cryptorchidism, hypospadias, varicocele or inguinal hernia, were also recorded. A second questionnaire was addressed to the parents of the volunteers and focused on questions related to periods close to conception, foetal life and birth of the subject. The transmission of the questionnaire to the parents was not a mandatory step. The option to fill out the questionnaires without participating in the clinical study was also given.

\section{Clinical Examination and Sample Collection}

A first clinical examination was performed for all conscripts during the medical check-up at the military camp. A series of parameters was recorded by the examiner, such as the testicular volumes measured with Prader's orchidometer, the anatomy of the genital area, the presence of surgical scars, hypospadias, cryptorchidism or varicocele. These clinical data were recorded anonymously without links to our studied population in order to get an overview of the general population. In this study, a cohort of 3210 subjects was analyzed and considered as the general population of young men.

A second examination was performed on the volunteers in our laboratory by our team physicians. The same parameters were recorded and an ultrasound examination was performed (Logiq $400 \mathrm{CL}, \mathrm{GE}$ Healthcare, Switzerland). This population was composed of 481 volunteers.

After clinical examination, blood, urine and sperm samples were collected from each volunteer. Semen samples were analysed immediately. All samples were aliquoted and stored in plastic tubes at minus $80^{\circ} \mathrm{C}$ (New Brunswick Scientific U535, IG Instrumenten-Gesellschaft, Switzerland) for further analysis.

\section{Semen Analysis}

Each volunteer provided a semen sample by masturbation in a room close to the laboratory. The ejaculate was collected into a sterile $15 \mathrm{ml}$ tube mounted with a plastic funnel (Spermo-Sampler, Veridial, Switzerland). The date and time of the last ejaculation were recorded in order to calculate precisely the period of sexual abstinence. Sperm volume was determined by weighing the collecting device before and after collection. Sperm samples were incubated for 30-60 min at $37{ }^{\circ} \mathrm{C}$ before analysis. A $5 \mu \mathrm{l}$ sperm aliquot was transferred in a 20 $\mu \mathrm{m}$ deep counting chamber (Leja Products, The Netherlands) and submitted to Computer Assisted Sperm Analysis (CASA, SCA System, Microptic SL, Spain). The CASA system recorded video sequences, which were stored and immediately analyzed in order to determine the concentration (in $10^{6} / \mathrm{ml}$ ) and various kinetic sperm parameters (e.g. WHO motility grades, average velocity, beat cross frequency). Smears were also prepared for morphologic examination after Papanicolaou staining. [19] The percentage of normal forms was determined under 1000-fold magnification (Eclipse 50i, Nikon, Switzerland) by two technicians trained in andrology. Two hundred spermatozoa were observed and scored as normal or abnormal according to the Kruger's criteria. ${ }^{[19]}$ A morphometric analysis was also performed with the CASA system according to the manufacturer's recommendations (SCA System, Microptic SL, Spain). Sizes (length, width, area, perimeter) and shapes (ellipticity, rugosity, elongation, regularity) of the sperm head as well as acrosome volume and midpiece size were recorded and stored. The morphometric normality of the spermatozoon was inferred using a computer algorithm based on the Kruger's strict criteria. All sequences, pictures and measurements could at all times be retrieved for further possible reanalysis. 


\section{Geographic Stratification}

In order to observe sperm quality variations in relation to the region of life or of gestation, data were stratified in different groups according to the classical Swiss major landscapes (Jura, Plateau and Alps). Moreover a sub-stratification was performed in the Plateau. One region, called 'West Plateau', was composed of the French-speaking part of the Plateau and the other, called 'Zürich', consisted of the cantons near Zürich city, the largest community in Switzerland. The stratification is represented in the Fig. At this stage, no data were available for the other regions of Switzerland, represented in white in the Fig.

\section{Anonymity and Transmission of Results}

In order to preserve anonymity, each subject received an identification code. The military medical supervisor of the study (ES) is the only person able to link the subject with his personal code. In our database and on all research documents, only the identification code was recorded. There was no possible retrieval of a personal identity, nor accidental release of such data to external parties.

During the enrolment phase, each volunteer was asked whether he wished to know the results of the sperm test. More than $99 \%$ of the volunteers declared an interest in receiving a report. Once the tests were performed, the following results were transmitted to the conscript through the army clinical supervisor: volume, concentration, total sperm count and percentage of motile forms. The WHO thresholds for each parameter were also given. A biological validation was then performed and four different possible comments were given:

i) All observed parameters are above the WHO reference values;

ii) One or several parameter(s) fall(s) slightly below the reference value(s);

iii) One or several parameter(s) fall(s) strongly below the reference value(s);

iv) No spermatozoa are present in the ejaculate.

Volunteers having comment iii or iv were invited to provide another sperm sample (visit the laboratory again) at least three months after the first test in order to confirm or invalidate the first result.

\section{Database and Statistics}

All the data collected were stored in an in-house built database (FileMaker Inc., Santa Clara, USA). Close to 800 variables were stored in each record. Data were then exported and studied using a commercially available statistical package (STATA Corp., Texas, USA).

Because most of the parameters are not normally distributed, it was decided to work with medians to express the sample estima-

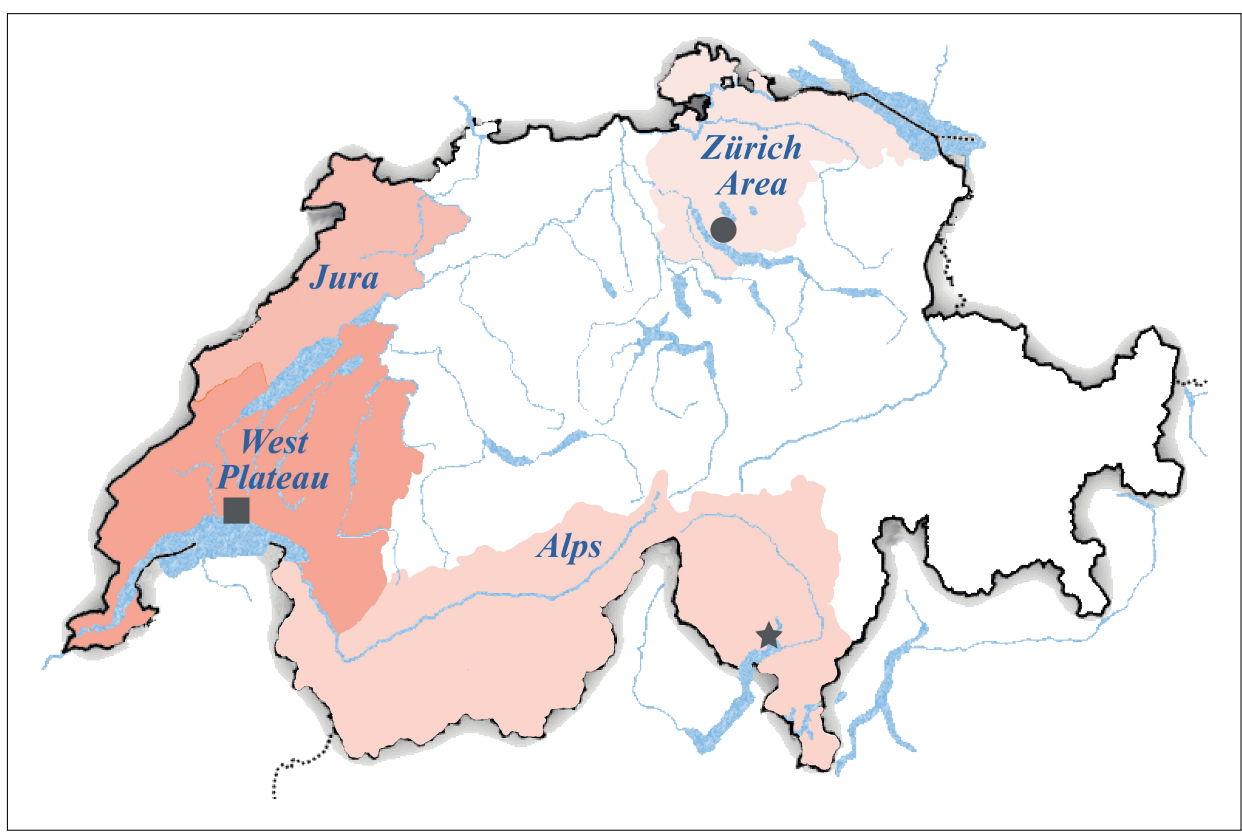

Fig. Geographic stratification according to the major Swiss landscapes: West Plateau, Jura, Alps and Zürich area. The colour density is related to the sperm concentration in each region. The grey square represents the main laboratory of Lausanne; the grey circle, the associated centre of Rüti and the grey star, the associated cente of Monte Ceneri.

tor as well as with the 10th and 90th percentiles to evaluate the sample dispersion. The statistic significances were estimated using the Kruskal-Wallis rank test for equality of populations or the Chi-square test for population independency. No confounding parameter was considered in the performed statistical analysis.

\section{Results}

\section{Participation Rate}

From September 2005 to June 2007, more than 19'000 young men were invited to participate in the military recruiting camps in three different recruiting centres (Lausanne, Rüti, and Monte Ceneri). During this period, 770 volunteers decided to participate in the complete study (questionnaires and clinical part), which represented a participation rate of about $4 \%$. From a cantonal perspective, the participation rate has considerably varied from a high $8.3 \%$ to a low $2.9 \%$.

\section{Physical and Health Characteristics of the Studied Population}

The studied population was highly homogenous as shown by the narrow $95 \%$ confidence intervals for physical parameters: age 19.8-20.0 years; self-reported height $178-179 \mathrm{~cm}$; self-reported weight 71-73 kg; BMI 22.4-22.9 kg/m². Each participant reported in the questionnaire his self-rated health (SRH). On a total of 680 men, 337 reported having a "very good' SRH (49.6\%); 324 (47.6\%), 'good' SRH; 19 (2.8\%), 'poor'SRH. The majority of the young volunteers $(97.2 \%)$ claimed to be healthy with reporting a good or very good SRH.

The general urogenital health of the conscripts was studied using three different approaches, which are the questionnaire filled out by the conscripts, the physical examination in the military recruiting centres, and the clinical examination at the research centres. For the time being, specific aspects suspected to affect spermatogenesis and which have been associated with environmental pollutions, ${ }^{[20]}$ have been quantified in the observed volunteer population $(n=481)$ and in the larger conscripts' population $(n=3,210)$ issued from military camp. The incidence of genital tract malformations in these two populations could be, respectively, reported as follow: varicocele $17.9 \%$ and $6.8 \%$; cryptorchidism $1.6 \%$ and $0.5 \%$; hydrocele $5.2 \%$ and $0.2 \%$; other malformations $14.8 \%$ and $7.0 \%$. The other malformations represented a range of rare malformations, which were grouped together in a unique category. In both populations, no testicular tumour was found. This comparison indicated significantly higher incidences of varicoceles $(\mathrm{p}<0.001)$, cryptorchidism ( $p$ $=0.005)$ and hydroceles $(\mathrm{p}<0.001)$ among the volunteers. All other genital tract malformations were comparable to those of the general population.

The mean total testis volumes, with 95\% confidence interval in square brackets, measured by orchidometry and ultrasonography were, respectively, $38.2 \mathrm{ml}$ [37.5-39.0 $\mathrm{ml}]$ and $22.3 \mathrm{ml}$ [21.6-23.0 ml]. The difference between the two methods $(15.9 \mathrm{ml})$, corresponding to $42 \%$ of orchidometrymeasured volume, was highly significant 
( $\mathrm{p}<0.0001)$. The testis size measured by orchidometry is comparable to that already published for young male populations. [21]

\section{Sperm Analysis}

Results of the semen analysis for the investigated cohort are summarized in Table 1. The median of sperm parameters is given as well as the 10th and 90th percentiles. These parameters are the sexual abstinence in days, the concentration in $10^{6}$ spermatozoa per millilitre, the total sperm count in $10^{6}$ spermatozoa per ejaculate, the percentage of motile forms and the percentage of morphologically ideal forms. The subjective morphology represents an analysis performed by trained technicians, while the objective morphology stands for the CASA method. The WHO reference values are also specified.

A comparison to a similar study performed in Scandinavian countries ${ }^{[21]}$ is summarized in Table 2. The median volume, the median sperm concentration and 5th/95th percentiles are given. The sperm concentration in Switzerland was in the same range of that reported by Jorgensen et al. ${ }^{[21]}$ More precisely, the values from Switzerland (47 $\times 10^{6} / \mathrm{ml}$ ) were below those from Norway $\left(53 \times 10^{6} / \mathrm{ml}\right)$ and close to those from Denmark $\left(44 \times 10^{6} / \mathrm{ml}\right)$.

\section{Geographic Stratification}

In order to minimize confounding effects, it was decided to restrict the observed cohort to the people reporting a sexual abstinence of 2-4 days. Thus, the geographic stratification was performed on data recorded on 329 cases representing $51 \%$ of the complete investigated volunteer population $(n=642)$. Table 3 shows the median sperm concentration, in million spermatozoa per millilitre, according to the region of residence or the region of gestation.

Looking at the region of residence, one could observe that two distinct groups emerged. The first one is composed of the Jura and the West Plateau and the second by the Alps and Zürich area. Statistically significant differences in the median sperm counts appeared between the four studied regions $(p=0.02)$. Similar variations were observed when analysing the data according to the regions of gestation $(p=0.03)$. The subjects who were conceived and born abroad had sperm counts close to those of the Jura/West Plateau group.

\section{Second Analysis}

On a total of 522 sperm samples, 235 (45\%) had all the measured parameters above the WHO reference values. In 164 cases, one or several parameters were slightly below these values and the volunteers were not invited to repeat the sperm test. It should be noticed that, strictly speaking, $55 \%$ of the observed cohort have one or

Table 1. Sperm parameters of the studied cohort $(n=653)$

$\begin{array}{lccrrr} & \text { Unit } & \begin{array}{c}\text { WHO } \\ \text { references }\end{array} & \text { Median } & \text { p10 } & \text { p90 } \\ \text { Abstinence } & {[\text { days }]} & 2-7 & 3.0 & 1.7 & 5.9 \\ \begin{array}{l}\text { Concentration } \\ \text { Volume }\end{array} & {\left[10^{6} / \mathrm{ml}\right]} & >20 & 47.0 & 10.2 & 137.5 \\ \begin{array}{l}\text { Total sperm } \\ \text { count }\end{array} & {[\mathrm{ml}]} & 2-6 & 2.9 & 1.4 & 5.0 \\ \begin{array}{l}\text { Sperm } \\ \text { motility }\end{array} & {[\% \text { ejaculate] }} & >40 & 131.5 & 22.0 & 422.4 \\ \begin{array}{l}\text { Subjective } \\ \text { morphology }\end{array} & n=255 & >50 & 58.2 & 34.2 & 81.6 \\ \begin{array}{l}\text { Objective } \\ \text { morphology }\end{array} & {[\%]} & >14 & 6.5 & 2.0 & 14.0 \\ & n=466 & >14 & 5.0 & 1.0 & 14.0\end{array}$

Table 2. Comparison of sperm concentrations between Switzerland and Scandinavian countries

\begin{tabular}{lcccc} 
& $\begin{array}{c}\text { Median volume } \\
{[\mathrm{ml}]}\end{array}$ & $\begin{array}{c}\text { Median concentration } \\
{[106 / \mathrm{ml}]}\end{array}$ & $\mathrm{p5}$ & $\mathrm{p95}$ \\
\hline Estonia $^{\mathrm{a}}$ & 3.1 & 62 & 13 & 175 \\
Finland $^{\mathrm{a}}$ & 3.0 & 61 & 3 & 184 \\
Norway $^{\mathrm{a}}$ & 2.9 & 53 & 7 & 179 \\
Switzerland & $\mathbf{2 . 9}$ & $\mathbf{4 7}$ & $\mathbf{4}$ & $\mathbf{1 7 3}$ \\
Denmark $^{\mathrm{a}}$ & 3.0 & 44 & 4 & 163 \\
\hline${ }^{\text {a }}$ From ref. [21] & & & &
\end{tabular}

Table 3. Median sperm concentration (in $10 \% / \mathrm{ml}$ ) according to region of living and gestation for a sexual abstinence of 2 to 4 days.

\begin{tabular}{lcccc} 
& \multicolumn{2}{c}{ Region of living } & \multicolumn{2}{c}{ Region of gestation } \\
\cline { 2 - 3 } & $n$ & $10^{6} / \mathrm{ml}$ & $n$ & $10^{6} / \mathrm{ml}$ \\
Abroad & \multicolumn{2}{c}{ no cases } & 21 & 55 \\
Jura & 75 & 54 & 76 & 54 \\
West Plateau & 169 & 54 & 150 & 53 \\
Alps & 46 & 43 & 40 & 40 \\
Zürich & 39 & 36 & 41 & 36 \\
Total & 329 & 50 & 328 & 50 \\
Kruskal-Wallis test & & 0.02 & & 0.03
\end{tabular}

several sperm parameters below the WHO reference values.

In 114 cases, the subjects were invited to repeat their sperm test because one or more parameters were clearly below the WHO references. In nine cases, no spermatozoa were found in the ejaculate and the subjects were asked to come back for confirmation. Out of these 123 cases, only 19 conscripts $(15.6 \%)$ responded positively to this invitation. Out of the 19 repeated tests, 12 subjects had an abnormal sperm concentration in the second samples. Azoospermia was confirmed in the two subjects who repeated the test.

\section{Discussion}

In this study, we present the first preliminary results on urogenital health and semen quality of Swiss young men. An observation of sperm concentration according to a regional classification is also introduced. To our knowledge, this is the first study performed in Switzerland with volunteers who are not patients undergoing a fertility treatment. It seems also to be the first time that we observe semen quality in regard to a geographic repartition in our country.

The mean participation rate $(4 \%, \mathrm{n}=$ $770)$ is extremely low regarding other com- 
parable studies performed in Scandinavian countries where the participation rates were between $13 \%$ and $19 \% .{ }^{[22]}$ For the moment, we do not have any indication to explain such a low participation. Nevertheless, we observed a cantonal discrepancy indicating a higher participation rate in rural regions than in urban ones.

The studied population is highly homogenous in term of age, height, weight and BMI. Although height and weight are self-reported, data are valuable because of the gender of the subjects. Males underestimate their weight less than females.[23] Moreover, each volunteer was weighed and measured the day before commencing the military camp.

Our studied population reported, using self-rated health (SRH), a good general health. SRH is a valuable marker for general health because it includes somatic and physiological health and is not only based on diseases but also on positive phenomena such as a high level of vitality and a good quality of life.[24]

The highest incidence of genital tract malformation was the presence of varicocele. It was the case for the volunteers' population (17.9\%) and for the larger group of conscripts $(6.8 \%)$. This last incidence is comparable to the one observed in the literature for young men. ${ }^{[25]}$ The higher incidence in the volunteers' group could be explained by use of ultrasonography and a better training of the urologists of the research team. A higher incidence of hydrocele could probably also be explained by the same mechanisms. In our study group, more than $5 \%$ of the subjects had an operated cryptorchid testis, whereas this rate was below $1 \%$ in the larger studied group in the recruitment centres. One can explain the higher incidence of cryptorchid volunteers by the fact that, being aware of the possible consequences of this condition on their fertility, they were more ready to participate in the study.

The absence of marked pathologies among the conscripts is probably falsely reassuring. In fact, testicular cancer is a rare disease with an incidence of 8.4/100'000 inhabitants in Switzerland. ${ }^{[26]}$ It is therefore not surprising that no cases were discovered in the study so far. Testicular dysgenesis syndrome (TDS) has been considered as an indicator for subtle links between decline of testicular function and increase in cancers of male gonads. ${ }^{[27,28]}$

The testicular volume observed in our studied cohort is comparable to the published values for both methods.[29] The difference between orchidometry and ultrasonography lies in the fact that the first method overestimates the testicular volume and the second underestimates it. [30]

The median value was above the WHO references for all parameters, except mor- phology. It should be noted that the 10th percentiles were clearly below these references for all investigated parameters. One could also observe the broad statistical dispersion of the concentration and the total sperm count.

A sperm concentration below $20 \times 10^{6} /$ $\mathrm{ml}$ is often considered as abnormal, because it lies below the reference value published by the WHO guidelines (WHO 1999). These guidelines are defined by a group of international experts who have studied populations of healthy fertile men. The reference values are not intended to set a limit below which men are infertile. It was reported that, with an increasing sperm concentration up to approximately $40 \times 10^{6} / \mathrm{ml}$, the waiting time to pregnancy of a couple decreases. ${ }^{[31]}$ In our studied population, up to $42 \%$ of the volunteers have a sperm concentration below this threshold. The future fatherhood or the eventual urogenital tract diseases of this cohort should be observed in ten years in order to evaluate the prospective aspects of the semen analysis on fertility.

The best controlled studies performed with a similar design to ours are those from Scandinavian countries. ${ }^{[21]}$ The median value of sperm concentration measured in Switzerland $\left(47 \times 10^{6} / \mathrm{ml}\right)$ comes close to the Danish one $\left(44 \times 10^{6} / \mathrm{ml}\right)$. It should be noted that in Europe, Denmark is known to have the highest prevalence of testicular cancers and TDS. ${ }^{[32]}$ An impaired spermatogenesis is part of the TDS, which has been related to environmental influences, including endocrine disruptors. ${ }^{[28,33]}$ One thus has to consider that the low testicular activity of the Swiss conscripts should be recognised as a national health issue, to be taken seriously and further investigated.

We have shown, in terms of sperm concentration, that statistical differences exist between regions in Switzerland. This observation can be linked to the one performed in two Nordic countries. Denmark has one of the highest testicular cancer incidences in Europe whereas Finland has one of the lowest. ${ }^{[34]}$ This distribution is also observed on the sperm concentration level $\left(41 \times 10^{6}\right)$ $\mathrm{ml}$ in Danish men versus $54 \times 10^{6} / \mathrm{ml}$ in Finnish men). [21] In our Swiss cohort, two regions present the same trend regarding sperm concentration. We reported, in the West Plateau, a concentration of $54 \times 10^{6} /$ $\mathrm{ml}$ whereas a density of $43 \times 10^{6} / \mathrm{ml}$ was observed in the Alps. Concerning the lowest concentration in the Zurich area, we have no clear explanation so far and this value has to be taken with caution. It cannot be excluded that another confounding factor, acting as an unsuspected bias, is interacting with our results. A larger study group will help to confirm these preliminary results.

More than $50 \%$ of the whole cohort of investigated subjects had a sperm sample below the WHO reference values. Despite such low semen quality, response to the second invitation for further analysis was poor and therefore no state-of-the-art conclusion could be drawn on the status of the whole cohort.

We report, for the first time, data regarding urogenital health and semen quality in a healthy Swiss population of young men. Although these men rated their health as good, the median sperm concentration was comparable to that of Denmark, a country known as presenting high TDS rates. Furthermore, low sperm parameters tend to influence future fecundity. This is worrying and should be recognised as a national health issue. The discrepancies emphasized between regions could be related to environmental factors, including endocrinedisruptor compounds. Thus, completion of the map of Switzerland in terms of sperm parameters and urogenital health is crucial: it should allow the study of the epidemiological, geographical and causal elements involved in the TDS.

\section{Acknowledgements}

The authors would like to thank the 'Praxis am Bahnhof' (Dr. Christoph Zeller) and the 'Laboratorio di Biologia della Riproduzione del Centro Cantonale di Fertilità' (Dr. Alessandra Fusconi) for managing volunteers in Rüti and in Monte Ceneri respectively. Acknowledgments go also to the Fertas Laboratories (Karine Besenval, Cadia Cataldo) for performing morphological sperm analysis. The authors would also acknowledge Dr. Silvio-Emil Djitik and Dr. Reza Sohani for performing clinical examinations in Lausanne as well as Dr. Marysa Emery for critical review of this manuscript. Finally, special thanks go to all volunteers participating to this study. This work was supported by Swiss National Science Foundation (NRP50, grant no. 4050-66564)

Received: March 22, 2008

[1] V. Axiak, A. J. Vella, D. Agius, P. Bonnici, G. Cassar, R. Casson, P. Chircop, D. Micallef, B. Mintoff, M. Sammut, Sci. Total Environ. 2000, 1, 89.

[2] L. J. Guillette, Jr., T. S. Gross, D. A. Gross, A. A. Rooney, H. F. Percival, Environ. Health Perspect. 1995, 103, 31.

[3] L. J. Guillette, Jr., Environ. Health Perspect. 2006, 114, 9.

[4] L. J. Guillette, Jr., T. S. Gross, G. R. Masson, J. M. Matter, H. F. Percival, A. R. Woodward, Environ. Health Perspect. 1994, 8,680 .

[5] M. R. Milnes, D. S. Bermudez, T. A. Bryan, M. P. Gunderson, L. J. Guillette, Jr., Biol. Reprod. 2005, 5, 1004.

[6] D. Bernet, T. Wahli, C. Kueng, H. Segner, Dis. Aquat. Organ. 2004, 1, 137.

[7] H. Schmidt-Posthaus, D. Bernet, T. Wahli, P. Burkhardt-Holm, Dis. Aquat. Organ. 2001, 3, 161 .

[8] J. G. Vos, E. Dybing, H. A. Greim, O. Ladefoged, C. Lambre, J. V. Tarazona, I. Brandt, A. D. Vethaak, Crit. Rev. Toxicol. 2000, 1,71 . 
[9] H. Ulfelder, Cancer 1980, 12, 3008.

[10] S. H. Swan, APMIS 2000, 12, 793.

[11] J. Toppari, J. C. Larsen, P. Christiansen, A. Giwercman, P. Grandjean, L. J. Guillette, Jr., B. Jegou, T. K. Jensen, P. Jouannet, N. Keiding, H. Leffers, J. A. McLachlan, O. Meyer, J. Muller, E. Rajpert-De Meyts, T. Scheike, R. Sharpe, J. Sumpter, N. E. Skakkebaek, Environ. Health Perspect. 1996, $104,741$.

[12] M. F. Costello, P. Sjoblom, Y. Haddad, S. J. Steigrad, E. G. Bosch, J. Assist. Reprod. Genet. 2002, 6, 284.

[13] S. H. Swan, E. P. Elkin, L. Fenster, Environ. Health Perspect. 2000, 10, 961.

[14] N. E. Skakkebaek, E. Rajpert-De Meyts, N. Jorgensen, E. Carlsen, P. M. Petersen, A. Giwercman, A. G. Andersen, T. K. Jensen, A. M. Andersson, J. Muller, APMIS 1998, 1, 3; discussion 12 .

[15] J. Auger, J. M. Kunstmann, F. Czyglik, P. Jouannet, N. Engl. J. Med. 1995, 5, 281.

[16] P. Jouannet, C. Wang, F. Eustache, T. Kold-Jensen, J. Auger, APMIS 2001, 5 , 333.

[17] L. Storgaard, J. P. Bonde, E. Ernst, M. Spano, C. Y. Andersen, M. Frydenberg, J. Olsen, Epidemiology 2003, 3, 278.

[18] S. H. Swan, C. Brazil, E. Z. Drobnis, F. Liu, R. L. Kruse, M. Hatch, J. B. Redmon, C. Wang, J. W. Overstreet, Environ. Health Perspect. 2003, 4, 414.

[19] WHO, 'Laboratory Manual for the Examination of Human Semen and SpermCervical Mucus Interaction', Cambridge University Press, Cambridge, 1999.

[20] R. M. Sharpe, D. S. Irvine, BMJ 2004, 7437, 447.

[21] N. Jorgensen, E. Carlsen, I. Nermoen, M. Punab, J. Suominen, A. G. Andersen, A. M. Andersson, T. B. Haugen, A. Horte, T. K. Jensen, O. Magnus, J. H. Petersen, M. Vierula, J. Toppari, N. E. Skakkebaek, Hum. Reprod. 2002, 8, 2199.
[22] T. K. Jensen, N. Jorgensen, M. Punab, T. B. Haugen, J. Suominen, B. Zilaitiene, A. Horte, A. G. Andersen, E. Carlsen, O. Magnus, V. Matulevicius, I. Nermoen, M. Vierula, N. Keiding, J. Toppari, N. E. Skakkebaek, Am. J. Epidemiol. 2004, 1, 49.

[23] B. Sherry, M. E. Jefferds, L. M. GrummerStrawn, Arch. Pediatr. Adolesc. Med. 2007, 12, 1154.

[24] T. K. Jensen, N. Jorgensen, C. Asklund, E. Carlsen, T. S. Kristensen, M. Holm, N. E. Skakkebaek, Fertil. Steril. 2007, 5, 1366.

[25] M. Nussinovitch, E. Greenbaum, J. Amir B. Volovitz, Arch. Pediatr. Adolesc. Med. 2001, 7, 855.

[26] E. Huyghe, T. Matsuda, P. Thonneau, $J$. Urol. 2003, 1, 5 .

[27] A. Giwercman, L. Rylander, L. Hagmar, Y. L. Giwercman, Int. J. Androl. 2006, 1, 291; discussion 304.

[28] N. E. Skakkebaek, Horm. Res. 2003, 49.

[29] H. Sakamoto, K. Saito, Y. Ogawa, H. Yoshida, Urology 2007, 1, 158.

[30] H. Sakamoto, K. Saito, M. Oohta, K. Inoue, Y. Ogawa, H. Yoshida, Urology 2007, 1, 152.

[31] J. P. Bonde, E. Ernst, T. K. Jensen, N. H. Hjollund, H. Kolstad, T. B. Henriksen, T. Scheike, A. Giwercman, J. Olsen, N. E. Skakkebaek, Lancet 1998, 352, 1172.

[32] I. A. Olesen, S. B. Sonne, C. E. HoeiHansen, E. Rajpert-DeMeyts, N. E. Skakkebaek, Best Pract. Res. Clin. Endocrinol. Metab. 2007, 3, 462.

[33] K. Bay, C. Asklund, N. E. Skakkebaek, A. M. Andersson, Best Pract. Res. Clin. Endocrinol. Metab. 2006, 1, 77.

[34] L. Richiardi, R. Bellocco, H. O. Adami, A. Torrang, L. Barlow, T. Hakulinen, M. Rahu, A. Stengrevics, H. Storm, S. Tretli, J. Kurtinaitis, J. E. Tyczynski, O. Akre, Cancer Epidemiol. Biomarkers Prev. 2004, 12, 2157. 